\title{
NASA's Evolution to Ka-band Space Communications for Near-Earth Spacecraft
}

Kevin McCarthy and Frank Stocklin

NASA Goddard Space Flight Center

Barry Geldzahler

NASA Headquarters

Daniel Friedman and Peter Celeste

Booz Allen Hamilton

SpaceOps 2010 - Huntsville, Alabama

April 27, 2010 


\section{Agenda}

$>$ Introduction and Reference Mission Requirements

$>$ Potential Solutions

$>$ Coverage and Loading Analysis

$>$ Recommended Solution

- Solution Options

- Proposed Space and Ground Assets

- Spectrum Plan

- Link Analysis

- Propagation Impairments

- Near-Earth Network (NEN) Ground Station Antennas Systems

- Wide Area Network (WAN)

$>$ Technology Considerations

> Future Considerations

$>$ Summary 


\section{Introduction}

> NASA has explored a Ka-band system design for near-Earth communications

> Motivations for Ka-band operation:

- Higher data rates (exceeding X-band spectrum capabilities)

- X-band spectrum crowding

> Multiple Ka-band missions flying today...

- Lunar Reconnaissance Orbiter (LRO)

- 100 Mbps through $18.3 \mathrm{~m}$ WS1 antenna at White Sands Complex (WSC)

- Advanced Land Observing Satellite (ALOS)

- $\quad 277.52$ Mbps through Tracking and Data Relay Satellite (TDRS) to WSC

- Solar Dynamics Observatory (SDO)

- 150 Mbps through dedicated $18.3 \mathrm{~m}$ assets at WSC

... But future missions will need higher data rates (1 Gbps+) and expanded infrastructures

- Evolution to Ka-band for near-Earth missions has been expected

- Ka-band capabilities will enable a new class of earth and space science missions

> Funding to begin formulation in FY2011 has been requested from the Space Communication and Navigation (SCaN) program office, the responsible program office at NASA Headquarters 


\section{Reference Mission Requirements}

> DESDynl, SWOT, and HyspIRI missions recommended by National Research Council's 2007 decadal survey have significant daily data volume requirements, motivating 1 Gbps+ downlinks

> These three missions served as references for developing a near-Earth Ka-band communications capability

> HysplRI's needs could be satisfied with a dual-polarization X-band solution

DESDynl
$\begin{aligned} & \text { To study geologic } \\ & \text { hazards and global } \\ & \text { environmental change } \\ & \text { Launch: } 2015 \text { - 2018+ }\end{aligned}$
Orbit:
- 760 km altitude
980 inclination
- Sun-synchronous (Dsc Node 1100)
Data Volume (w/ Compression):
- 40 Tbits of data per day
Contact Requirements (@ 1 Gbps):
- 667 minutes per day
- 45 minutes per orbit

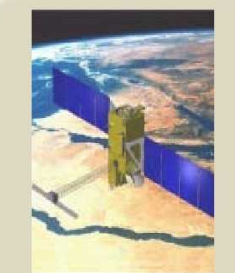

Orbit:

- $970 \mathrm{~km}$ altitude

- $78^{\circ}$ inclination

Data Volume (w/ Compression):

- 7.2 Tbits of data per day

Contact Requirements (@ 1 Gbps):

- 120 minutes per day

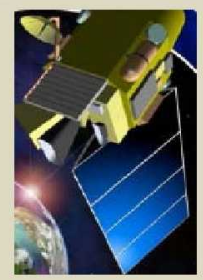

\section{HyspIRI}

To study global surface reflectance, temperature, and emissivity

Launch: 2015 - 2020+

Orbit:

- $626 \mathrm{~km}$ altitude

- $98^{\circ}$ inclination

- Sun-synchronous (Asc Node 1800)

Data Volume (w/ Compression):

- 3.5 Tbits of data per day

Contact Requirements (@ 1 Gbps):

- 60 minutes per day

\section{Additional Potential Ka-band Missions}

$\begin{array}{cccccccc}\text { GEO-CAPE } & \text { ACE } & \text { VEGBIOM } & \text { LEOMAC } & \text { PATH } & \text { SCLP } & \text { COPS } & \text { Future HSF } \\ \text { GEOMAC } & \text { ACE-Core } & \text { ACOB-A } & \text { LIST } & \text { GRACE-II } & \text { EXIST } & \text { GACM } & \text { Future Suborbital }\end{array}$


NASA

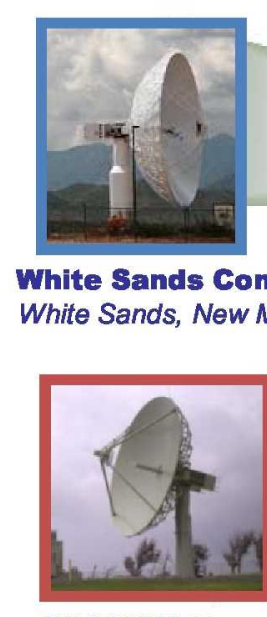

SSC/USN Hawail

South Point, Hawaii

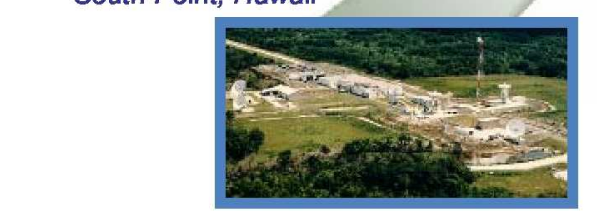

\section{NASA}

Commercial
- Partner

Merritt Island

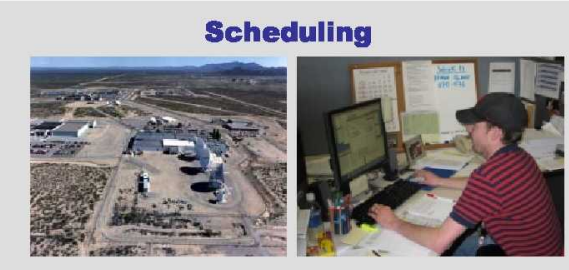

\section{Launch Annex}

Merritt Island, Florida plex

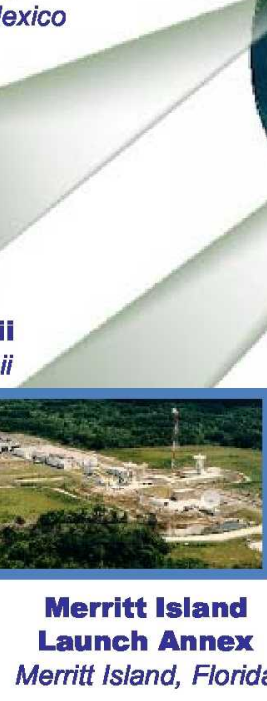

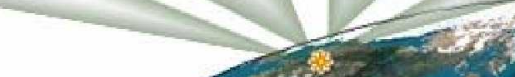

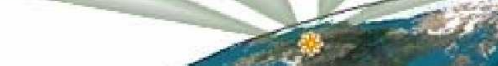
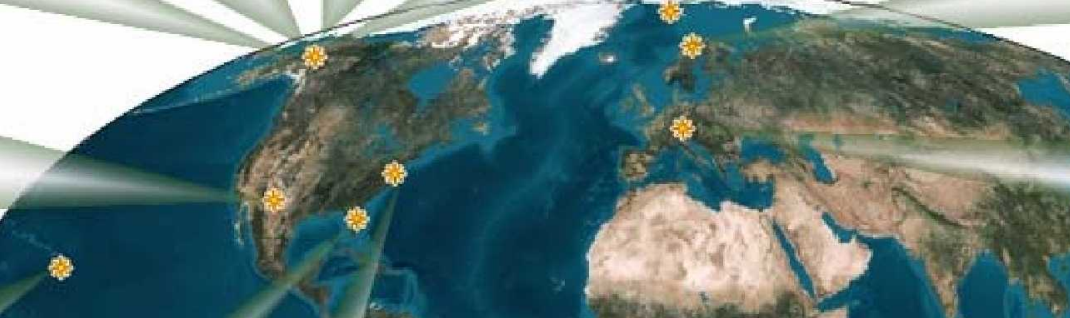

sa Wallops, Virginia

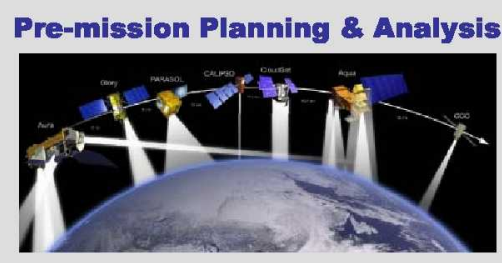

NASA Near Earth Network (NEN)
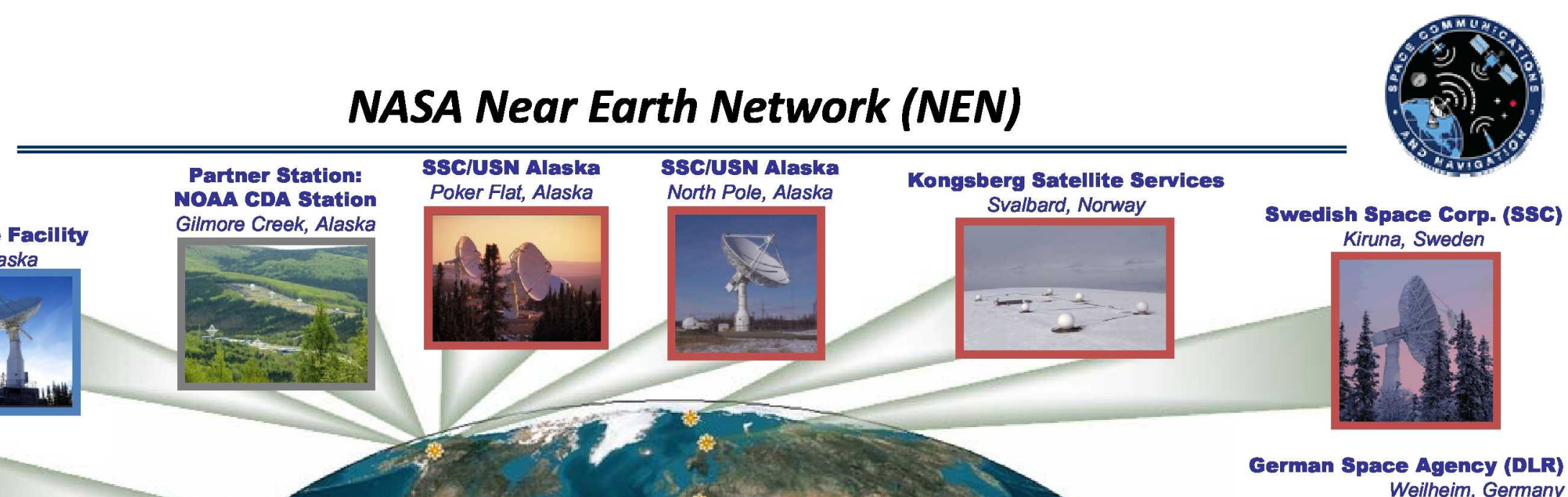

Cerman Space Agency (DLR) Weilheim, Germany
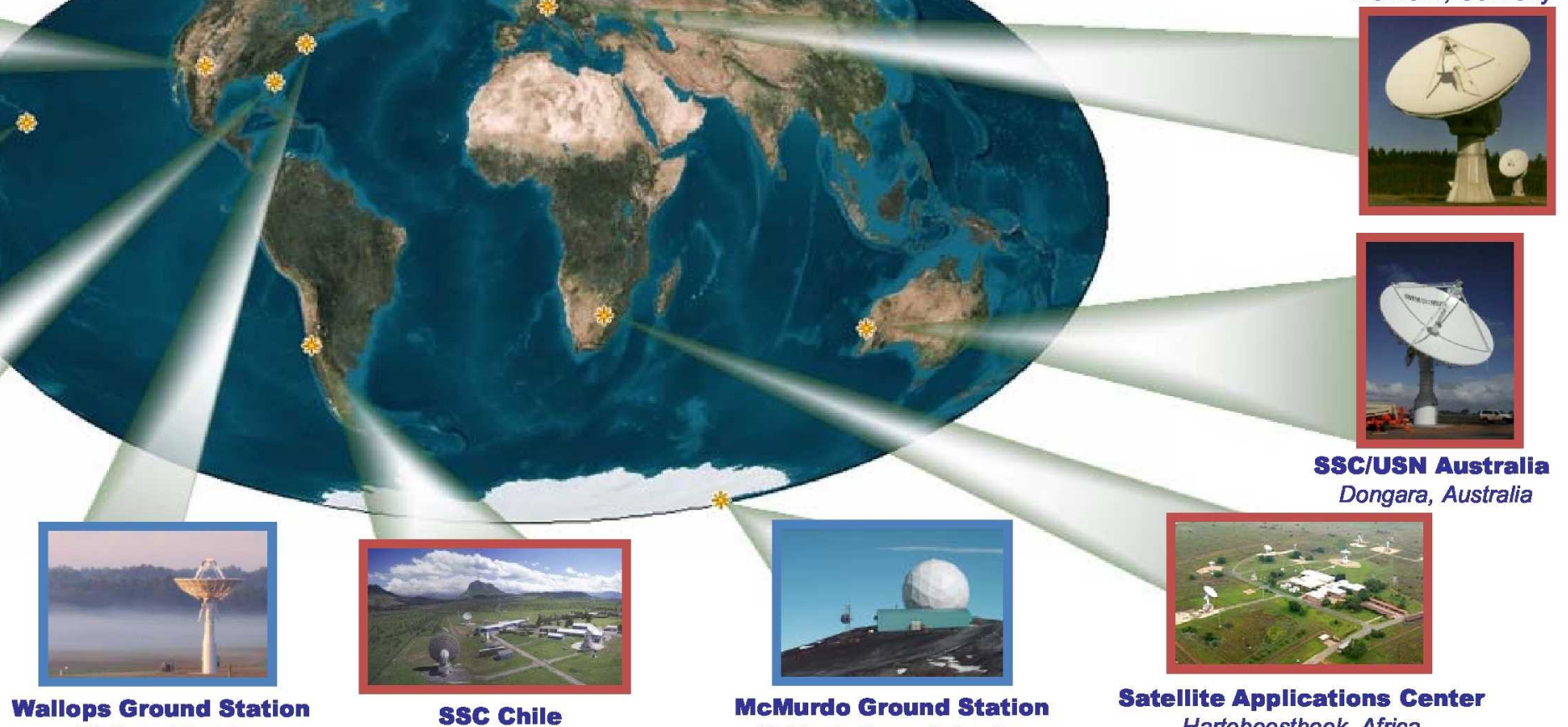
McMurdo Base, Antarctica

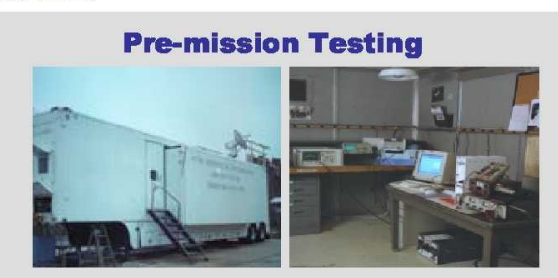

Network Monitoring \& Coordination

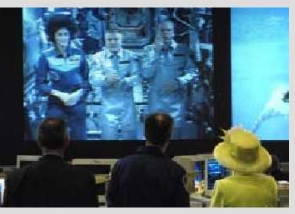

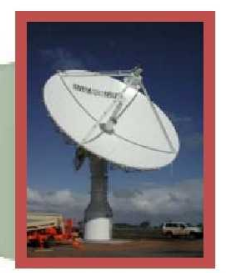

SSC/USN Australia Dongara, Australia

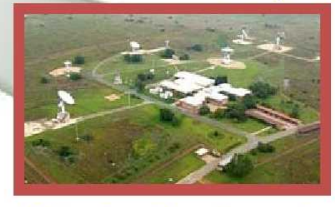

Satellite Applications Center Hartebeesthoek, Africa

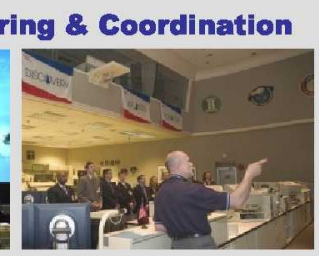




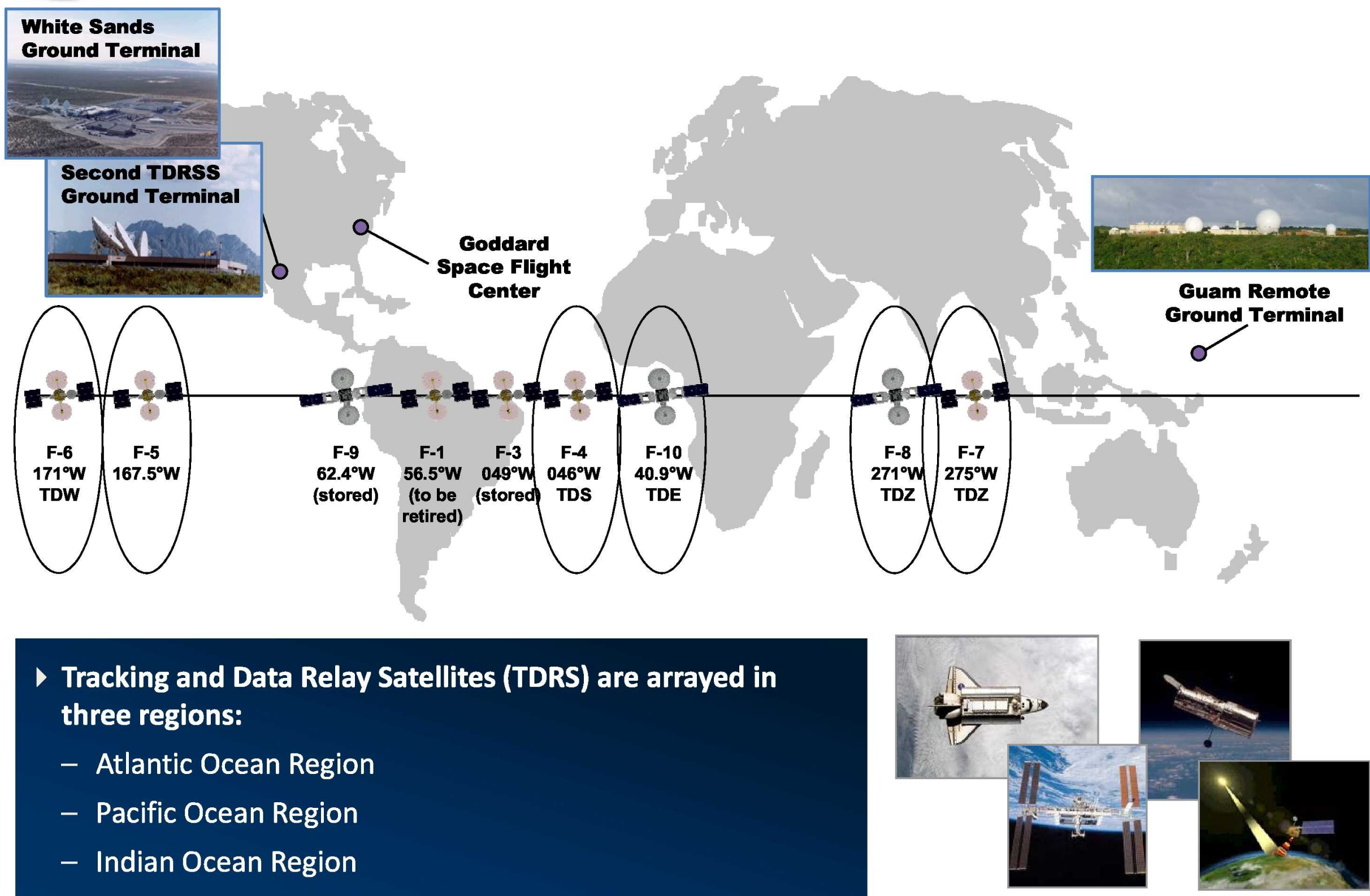




\section{Potential Solutions}

\begin{tabular}{|c|c|}
\hline Option & Considerations \\
\hline $\begin{array}{l}\text { NASA } \\
\text { Near Earth Network } \\
\text { (NEN) }\end{array}$ & $\begin{array}{l}\text { - Leverage existing "WS1" } 18.3 \mathrm{~m} \text { Ka-band asset at WSC with upgrades } \\
\text { - Entails establishing new Ka-band assets and integrating into NEN } \\
\text { - Locations for new assets to be determined by multiple criteria (coverage, rain } \\
\text { attenuation, operational costs, backhaul costs, existing infrastructure, etc.) }\end{array}$ \\
\hline $\begin{array}{l}\text { NASA } \\
\text { Space Network } \\
\text { (SN) }\end{array}$ & $\begin{array}{l}\text { - Leverage Ka-capable satellites: } \\
\quad \text { - TDRS-8/9/10 (on-orbit) } \\
\quad \text { - TDRS-K/L (on-order, to be launched 4/2012 and 2/2013, respectively) } \\
\text { 1-Node Solution } \\
\text { - Risk-reduction/fallback solution from 2-node solution, to mitigate against } \\
\text { TDRS launch slip/failure or SN upgrade schedule slips } \\
\text { 2-Node Solution } \\
\text { - Greater coverage than 1-node solution } \\
\text { - Avoids costly WAN bandwidth upgrade from Guam (required for 3-node solution) } \\
\text { 3- Node Solution } \\
\text { - Worth considering if 2-node solution would not satisfy mission requirements } \\
\text { - Would require potentially cost-prohibitive WAN bandwidth upgrade from Guam }\end{array}$ \\
\hline $\begin{array}{l}\text { NOAA NPOESS } \\
\text { SafetyNet }\end{array}$ & $\begin{array}{l}\text { - Leverage } 15 \text {-station Ka-band infrastructure investment for operational weather } \\
\text { satellites by sharing capacity between NOAA and NASA }\end{array}$ \\
\hline
\end{tabular}




\section{SafetyNet}

> NOAA's National Polar-orbiting Operational Environmental Satellite System (NPOESS) SafetyNet network of 15 Ka-capable ground stations potentially offers a large infrastructure to support earth and space science missions

- NPOESS is being reformulated to support planned new Joint Polar Satellite System (JPSS)

Constraints:

- Small asset size $(4 \mathrm{~m})$ reduces $\mathrm{G} / \mathrm{T}(\sim 10 \mathrm{~dB})$ vs. $12 \mathrm{~m}$ antennas proposed in alternative solution

- Would require redesigning SWOT spacecraft antenna system

- 150 Mbps receivers would require upgrades to support 1 Gbps

- Limited backhaul connectivity ( $45 \mathrm{Mbps}$ )

- Scheduling conflicts with primary SafetyNet mission constrain availability for NASA missions

- Limited expansibility

- Cannot supply sufficient contact time for DESDynl (667 minutes/day)

- Even with all 15 stations and 1 Gbps downlink rate (!) 


\section{Options: Mission Support and Recommendation}

\begin{tabular}{|c|c|c|c|c|}
\hline \multirow[b]{2}{*}{ Option } & \multicolumn{3}{|c|}{ Mission Support } & \multirow[b]{2}{*}{ Other Considerations } \\
\hline & DESDynl & HyspIRI & SWOT & \\
\hline NEN & $\begin{array}{l}\text { Would require } \\
\text { unreasonably large } \\
\text { number of stations }\end{array}$ & $\begin{array}{l}\text { Mission } \\
\text { requirements can be } \\
\text { met with } 2 \text { or more } \\
\text { Ka-band ground } \\
\text { systems }\end{array}$ & $\begin{array}{l}\text { Mission } \\
\text { requirements can be } \\
\text { met with } 2 \text { or more } \\
\text { Ka-band ground } \\
\text { systems }\end{array}$ & $\begin{array}{l}\text { If DESDynl launch should slip past 2015, } \\
\text { a } 3 \text { Gbps solution could potentially be } \\
\text { used to meet mission requirements }\end{array}$ \\
\hline SN & $\begin{array}{l}\text { 2-node and 1-node } \\
\text { solutions could } \\
\text { satisfy requirements }\end{array}$ & $\begin{array}{l}\text { SN can satisfy } \\
\text { mission } \\
\text { requirements, } \\
\text { although a NEN } \\
\text { solution can do so } \\
\text { more easily }\end{array}$ & $\begin{array}{l}\text { Need to minimize } \\
\text { antenna size to limit } \\
\text { spacecraft jitter } \\
\text { induced by antenna } \\
\text { movement }\end{array}$ & $\begin{array}{l}\text { - Large burden on spacecraft vs. ground } \\
\text { - More expensive than ground solution }\end{array}$ \\
\hline $\begin{array}{l}\text { NPOESS } \\
\text { SafetyNet }\end{array}$ & $\begin{array}{l}\text { Does not satisfy } \\
\text { daily contact } \\
\text { requirement }\end{array}$ & $\begin{array}{l}\text { Requires upgraded } \\
\text { receivers, ground } \\
\text { connectivity }\end{array}$ & $\begin{array}{l}\text { Impact to S/C } \\
\text { antenna system; } \\
\text { requires upgraded } \\
\text { receivers, ground } \\
\text { connectivity }\end{array}$ & $\begin{array}{l}\text { - Schedule conflicts with primary } \\
\text { SafetyNet mission limit availability } \\
\text { for NASA missions } \\
\text { - Limited expansibility }\end{array}$ \\
\hline \multirow[t]{2}{*}{ Recommendation: } & Yes & Possible & No & \\
\hline & $\begin{array}{r}\text { Recommendatic } \\
\text { Cost-e }\end{array}$ & $\begin{array}{l}\text { n: Apply NEN an } \\
\text { ectively leverag } \\
\text { and offers max }\end{array}$ & $\begin{array}{l}\text { N as best suited } \\
\text { each network's si } \\
\text { um extensibility }\end{array}$ & $\begin{array}{l}\text { for each mission } \\
\text { rengths }\end{array}$ \\
\hline
\end{tabular}




\section{Coverage and Loading Analysis}

SN Analysis Summary for DESDynl

\begin{tabular}{ccc}
\hline SN Support Scenario & DESDynI Priority & $\begin{array}{c}\text { Contact time } \\
\text { (average } \\
\text { minutes/day) }\end{array}$ \\
\hline 2 satellites (TDE, TDW) & $<$ HST, Terra & 668 \\
1 satellite (TDE) & $>$ HST, Terra & 691 \\
\hline 1 satellite (TDE) & $<$ HST, Terra & 640 \\
\hline Mission Requirement & & 667 \\
\hline
\end{tabular}

HST = Hubble Space Telescope.

Nominal spacecraft antenna configuration assumed for these approximate results.

Potential second antenna aboard spacecraft, for ground terminal operation, was not included.
NEN Analysis Summary for SWOT, HyspIRI

\begin{tabular}{lcc}
\hline \multicolumn{1}{c}{ NEN Station } & \multicolumn{2}{c}{$\begin{array}{c}\text { Contact time per mission } \\
\text { (average minutes/day) }\end{array}$} \\
SWOT & HyspIRI \\
\hline Alaska & 51.9 & 21.2 \\
Svalbard & 55.1 & 27.5 \\
Wallops & 6.9 & 13.3 \\
White Sands & 16.6 & 7.9 \\
McMurdo & 66.6 & 55.7 \\
\hline Total & 197.1 & 125.6 \\
\hline Mission & 120 & 60 \\
\hline Requirements & & \\
\hline
\end{tabular}

Coverage calculations have been adjusted for overlap. Alaska and Svalbard together suffice for both missions' requirements, assuming optimized scheduling.

Figures for McMurdo assume SWOT and HyspIRI receive highest priority.

> Analysis results are based on expected orbital parameters

> Required contact times could be reduced with data rates greater than $1 \mathrm{Gbps}$

\section{Analysis confirms viability of SN-based solution for DESDynl and NEN-based solution for SWOT and HyspIRI}




\section{Recommended Solution: \\ Proposed Ka-band Assets}

\section{Location Selection Criteria}

$>$ Coverage

$>$ Rain attenuation

$>$ Operational costs

$>$ Backhaul costs

$>$ Existing infrastructure

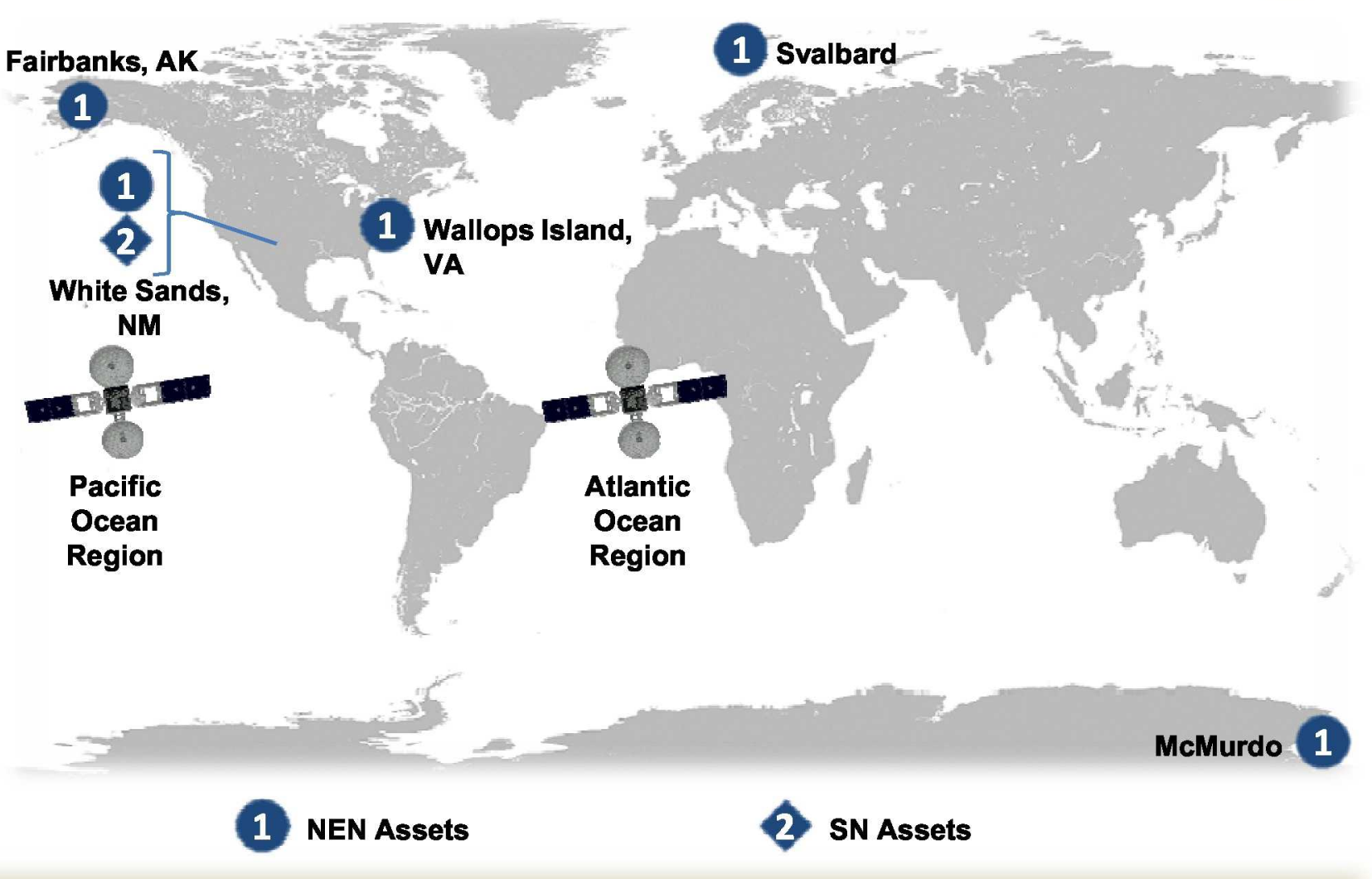

\section{Asset Information}

> SN assets:

- 2 TDRS spacecraft in Atlantic and Pacific Ocean Regions supported by WSC

$>5$ NEN assets:

- $12+m$ assets (new) at Alaska, Wallops, Svalbard

- $18.3 \mathrm{~m}$ WS1 asset (existing) at White Sands, upgraded to accommodate higher data rates

- $5.4 \mathrm{~m}$ asset (under development) at McMurdo, with additional back-end equipment to support higher data rates, and refurbished McMurdo-TDRS Relay System 2 (MTRS-2) link 


\section{Recommended Solution: Proposed Ka-band Spectrum Plan}

$25.25 \mathrm{GHz}$

$27.5 \mathrm{GHz}$

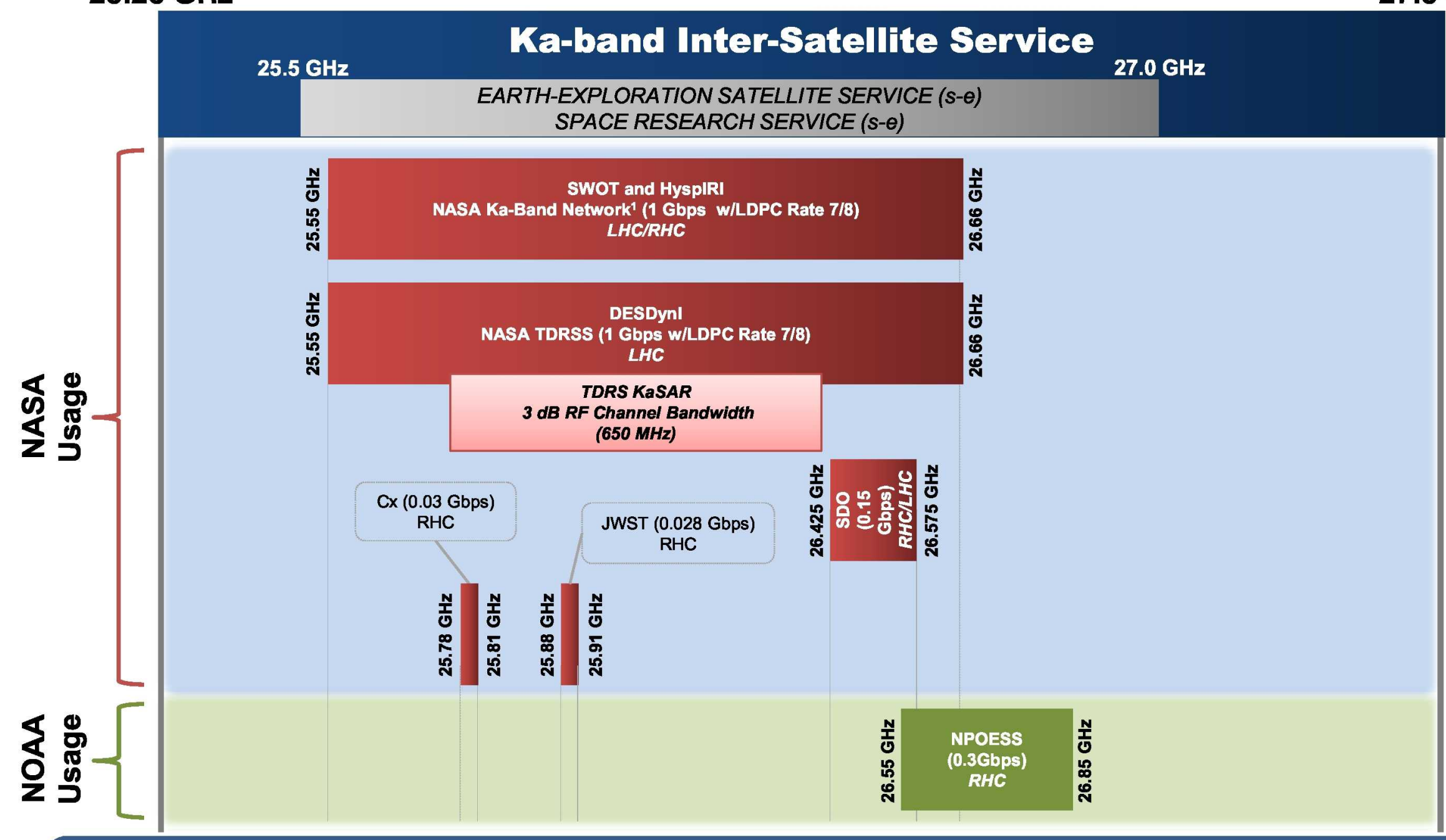

Proposed Ka-band spectrum plan provides NASA missions with adequate protection from interference with NPOESS. Potential interference between NASA Ka-band missions (e.g., SDO, Cx, JWST) can be accommodated through scheduling

${ }^{1}$ The proposed NASA Ka-band Network will procure antennas that have dual polarization capability, allowing for both LHC and RHC. 


\section{Recommended Solution: \\ Link Analysis Summary}

\begin{tabular}{|c|c|c|c|c|c|c|}
\hline Mission & SN & $\begin{array}{l}\text { Alaska } \\
(12 \mathrm{~m})\end{array}$ & $\begin{array}{l}\text { Svalbard } \\
(12 \mathrm{~m})\end{array}$ & $\begin{array}{c}\text { Wallops } \\
(12 \mathrm{~m})\end{array}$ & $\begin{array}{c}\text { WS1 } \\
(18.3 m)\end{array}$ & $\begin{array}{l}\text { McMurdo } \\
\text { (5.4m) }\end{array}$ \\
\hline $\begin{array}{l}\text { DESDynI } \\
\text { (1 Gbps, OQPSK, Rate-7/8 LDPC) } \\
\quad-\text { EIRP to SN }=60.3 \mathrm{dBW}\end{array}$ & $\begin{array}{l}M=2 d B \\
A A=99 \%\end{array}$ & N/A & N/A & N/A & N/A & N/A \\
\hline $\begin{array}{l}\text { SWOT } \\
\text { (1 Gbps, OQPSK, Rate-7/8 LDPC) } \\
\quad-\text { EIRP to NEN }=28.8 \mathrm{dBW}\end{array}$ & N/A & $\begin{array}{c}M=3 \mathrm{~dB} \\
A A=99 \% \\
E A=10 \mathrm{deg}\end{array}$ & $\begin{array}{c}M=4.7 \mathrm{~dB} \\
A A=99 \% \\
E A=10 \mathrm{deg}\end{array}$ & $\begin{array}{c}M=3 \mathrm{~dB} \\
A A=95 \% \\
E A=12.1 \mathrm{deg}\end{array}$ & $\begin{array}{c}M=4.9 \mathrm{~dB} \\
A A=99 \% \\
E A=10 \mathrm{deg}\end{array}$ & $\begin{array}{c}M=3 \mathrm{~dB} \\
A A=99 \% \\
E A=17.5 \mathrm{deg}\end{array}$ \\
\hline $\begin{array}{l}\text { HyspIRI } \\
\text { (1 Gbps, OQPSK, Rate-7/8 LDPC) } \\
\quad-\text { EIRP to NEN }=26.2 \mathrm{dBW}\end{array}$ & N/A & $\begin{array}{c}M=3 \mathrm{~dB} \\
A A=99 \% \\
E A=10 \mathrm{deg}\end{array}$ & $\begin{array}{c}M=4.7 \mathrm{~dB} \\
A A=99 \% \\
E A=10 \mathrm{deg}\end{array}$ & $\begin{array}{c}M=3 \mathrm{~dB} \\
A A=95.3 \% \\
E A=12 \mathrm{deg}\end{array}$ & $\begin{array}{c}M=4.9 \mathrm{~dB} \\
\mathrm{AA}=99 \% \\
E A=10 \mathrm{deg}\end{array}$ & $\begin{array}{c}M=3 \mathrm{~dB} \\
A A=99 \% \\
E A=16 \mathrm{deg}\end{array}$ \\
\hline
\end{tabular}

- Ground-based solution would require unreasonably many antennas $(>15)$ to provide sufficient contact time

- TDRS solution can support required contact time

> SWOT, HyspIRI:

- Link closure to TDRS deemed infeasible

- NEN-based solution suffices

- NEN availability target reduced and/or elevation angle increased for Wallops and McMurdo sites to obtain desired $3 \mathrm{~dB}$ link margin

\section{Combination of SN and proposed NEN Ka-band network can satisfy mission requirements with viable RF link designs}




\section{Recommended Solution: Propagation Impairments}

$>$ Ka-band propagation impairments include rain attenuation, cloud attenuation, gaseous absorption, and scintillation fading

$>$ New Ka-band NEN antennas will be designed to support orthogonal polarizations, so cross-polar discrimination (XPD)-a measure of the polarization isolation-is a concern as well

$>$ Literature survey and analysis were conducted to investigate these phenomena. Analysis results (@ $26 \mathrm{GHz}$; based on ITU-R Recommendation P.618-9):

\begin{tabular}{|c|c|c|c|c|c|c|c|c|c|c|c|c|c|c|c|c|}
\hline \multirow{2}{*}{\multicolumn{2}{|c|}{ Ground Station Information }} & \multicolumn{15}{|c|}{ Propagation Impairments for Percent Avallability } \\
\hline & & \multicolumn{5}{|c|}{$95 \%$} & \multicolumn{5}{|c|}{$97 \%$} & \multicolumn{5}{|c|}{$99 \%$} \\
\hline $\begin{array}{c}\text { Name, Latitude, } \\
\text { Longitude, Height } \\
\text { above MSL }\end{array}$ & $\begin{array}{l}\text { Elevation } \\
\text { Angle }\left({ }^{\circ}\right)\end{array}$ & $\begin{array}{l}\text { Rain } \\
\text { Atten. } \\
\text { (dB) }\end{array}$ & $\begin{array}{l}\text { Gas } \\
\text { Abs. } \\
\text { (dB) }\end{array}$ & $\begin{array}{l}\text { Scint. } \\
\text { Fade } \\
\text { (dB) }\end{array}$ & $\begin{array}{l}\text { Total } \\
\text { Fade } \\
\text { (dB) }\end{array}$ & $\begin{array}{l}\text { XPD } \\
(\mathrm{dB})\end{array}$ & $\begin{array}{l}\text { Rain } \\
\text { Atten. } \\
\text { (dB) }\end{array}$ & $\begin{array}{l}\text { Gas } \\
\text { Abs. } \\
\text { (dB) }\end{array}$ & $\begin{array}{l}\text { Scint. } \\
\text { Fade } \\
\text { (dB) }\end{array}$ & $\begin{array}{l}\text { Total } \\
\text { Fade } \\
(\mathrm{dB})\end{array}$ & $\begin{array}{l}\text { XPD } \\
\text { (dB) }\end{array}$ & $\begin{array}{l}\text { Rain } \\
\text { Atten. } \\
\text { (dB) }\end{array}$ & $\begin{array}{l}\text { Gas } \\
\text { Abs. } \\
\text { (dB) }\end{array}$ & $\begin{array}{l}\text { Scint. } \\
\text { Fade } \\
\text { (dB) }\end{array}$ & $\begin{array}{l}\text { Total } \\
\text { Fade } \\
\text { (dB) }\end{array}$ & $\begin{array}{l}\text { XPD } \\
\text { (dB) }\end{array}$ \\
\hline \multirow{4}{*}{$\begin{array}{c}\text { Fairbanks, AK } \\
65.974^{\circ} \mathrm{N} \\
147.512^{\circ} \mathrm{W} \\
549.0 \mathrm{~m}\end{array}$} & 5 & 1.32 & 1.82 & 0.83 & 3.38 & 32.51 & 1.92 & 1.82 & 1.00 & 3.99 & 29.92 & 4.06 & 1.82 & 1.36 & 6.10 & 24.56 \\
\hline & 10 & 0.77 & 0.95 & 0.29 & 1.77 & 36.97 & 1.14 & 0.95 & 35 & 2.13 & 34.36 & 2.44 & 0.95 & 0.48 & 3.44 & 28.96 \\
\hline & 15 & 0.57 & 0.64 & 0.15 & 1.23 & 39.70 & 0.84 & 0.64 & 18 & 1.50 & 37.09 & 1.83 & 0.64 & 0.25 & 2.48 & 31.67 \\
\hline & 20 & 0.46 & 0.48 & 0.10 & 0.96 & 41.77 & 0.68 & 0.48 & 0.12 & 1.18 & 39.15 & 1.50 & 0.48 & 0.16 & 1.99 & 33.72 \\
\hline \multirow{4}{*}{$\begin{array}{c}\text { Svalbard, Norway } \\
78.23072^{\circ} \mathrm{N} \\
15.3896^{\circ} \mathrm{E} \\
466.3 \mathrm{~m}\end{array}$} & 5 & 0.71 & 1.87 & 0.83 & 2.96 & 37.54 & 1.04 & 1.87 & 1.00 & 3.31 & 34.93 & 2.24 & 1.87 & 1.37 & 4.49 & 29.51 \\
\hline & 10 & 0.40 & 0.97 & 0.29 & 1.46 & 42.27 & 0.59 & 0.97 & 0.35 & 1.66 & 39.64 & 1.31 & 0.97 & 0.48 & 2.36 & 34.18 \\
\hline & 15 & 0.29 & 0.65 & 0.15 & 0.98 & 45.17 & 0.43 & 0.65 & 0.19 & 1.12 & 42.53 & 0.96 & 0.65 & 0.25 & 1.65 & 37.05 \\
\hline & 20 & 0.23 & 0.50 & 0.10 & 0.75 & 47.35 & 0.34 & 0.50 & 0.12 & 0.86 & 44.71 & 0.78 & 0.50 & 0.16 & 1.29 & 39.22 \\
\hline \multirow{4}{*}{$\begin{array}{c}\text { White Sands, NM } \\
32.5425^{\circ} \mathrm{N} \\
106.6121^{\circ} \mathrm{W} \\
1485 \mathrm{~m}\end{array}$} & 5 & 3.18 & 2.08 & 0.83 & 5.37 & 25.49 & 4.56 & 2.08 & 1.00 & 6.74 & 22.93 & 9.28 & 2.08 & 1.37 & 11.46 & 17.66 \\
\hline & 10 & 1.88 & 1.07 & 0.26 & 2.97 & 29.86 & 2.72 & 1.07 & 0.32 & 3.81 & 27.29 & 5.65 & 1.07 & 0.44 & 6.74 & 21.97 \\
\hline & 15 & 1.40 & 0.72 & 0.13 & 2.13 & 32.49 & 2.03 & 0.72 & 0.16 & 2.76 & 29.91 & 4.28 & 0.72 & 0.22 & 5.01 & 24.58 \\
\hline & 20 & 1.15 & 0.55 & 0.08 & 1.7 & 34.45 & 1.68 & 0.55 & 0.1 & 2.23 & 31.86 & 3.56 & 0.55 & 0.13 & 4.11 & 26.52 \\
\hline \multirow{4}{*}{$\begin{array}{c}\text { Wallops Island, } \\
\text { VA } 37.9235^{\circ} \mathrm{N} \\
75.4761^{\circ} \mathrm{W} \\
4.3 \mathrm{~m}\end{array}$} & 5 & 4.12 & 4.02 & 1.45 & 8.40 & 23.42 & 5.87 & 4.02 & 1.75 & 10.15 & 20.87 & 11.85 & 4.02 & 2.39 & 16.11 & 15.62 \\
\hline & 10 & 2.54 & 2.07 & 0.51 & 4.66 & 27.46 & 3.65 & 2.07 & 0.62 & 5.77 & 24.90 & 7.49 & 2.07 & 0.85 & 9.61 & 19.61 \\
\hline & 15 & 1.93 & 1.40 & 0.27 & 3.34 & 29.93 & 2.78 & 1.40 & 0.33 & 4.20 & 27.37 & 5.78 & 1.40 & 0.44 & 7.20 & 22.06 \\
\hline & 20 & 1.60 & 1.06 & 0.17 & 2.67 & 31.80 & 2.32 & 1.06 & 0.20 & 3.39 & 29.23 & 4.86 & 1.06 & 0.28 & 5.92 & 23.92 \\
\hline \multirow{4}{*}{\begin{tabular}{|c|} 
McMurdo, Antartica \\
$77.83913^{\circ} \mathrm{S}$ \\
$193.333^{\circ} \mathrm{E} \quad 206.4$ \\
$\mathrm{~m}$
\end{tabular}} & 5 & 0.02 & 1.28 & 0.78 & 2.06 & 65.16 & 0.03 & 1.28 & 0.94 & 2.22 & 62.42 & 0.09 & 1.28 & 1.29 & 2.57 & 56.69 \\
\hline & 10 & 0.01 & 0.67 & 0.31 & 0.98 & 71.73 & 0.02 & 0.67 & 0.38 & 1.05 & 68.96 & 0.04 & 0.67 & 0.52 & 1.19 & 63.16 \\
\hline & 15 & 0.01 & 0.45 & 0.18 & 0.64 & 75.56 & 0.01 & 0.45 & 0.22 & 0.67 & 72.79 & 0.03 & 0.45 & 0.30 & 0.76 & 66.95 \\
\hline & 20 & 0.00 & 0.34 & 0.12 & 0.47 & 78.35 & 0.01 & 0.34 & 0.15 & 0.49 & 75.57 & 0.02 & 0.34 & 0.20 & 0.55 & 69.72 \\
\hline
\end{tabular}

\section{Key conclusions:}

$>$ Careful attention must be paid to the axial ratios of both the transmitting and receiving antennas

$>$ Snow accumulation on radomes should be reasonably managed but does not significantly reduce polarization isolation

$>$ Ice particles and freezing rain can reduce polarization isolation 


\section{Recommended Solution: Ka-band NEN Ground Station Antenna Systems}

> June 2009 Request for Information (RFI):

\section{RFI Proposed Antenna System Configuration}

- Obtain technical and costing information from industry in response to draft Ka-Band antenna system requirements

$>$ Key antenna system requirements (based on link calculations):

- Aperture size $>=12 \mathrm{~m}$

- Continuously track LEO satellites $>=500 \mathrm{~km}$ above the earth

- Ka-band:

- Simultaneous LHCP and RHCP receive

- $\mathrm{G} / \mathrm{T}: 42.7 \mathrm{~dB} /{ }^{\circ} \mathrm{K}$

- S-Band:

- Simultaneous LHCP and RHCP receive

- $\mathrm{G} / \mathrm{T}: 23 \mathrm{~dB} /{ }^{\circ} \mathrm{K}$

- Transmit EIRP: $63 \mathrm{dBW}$

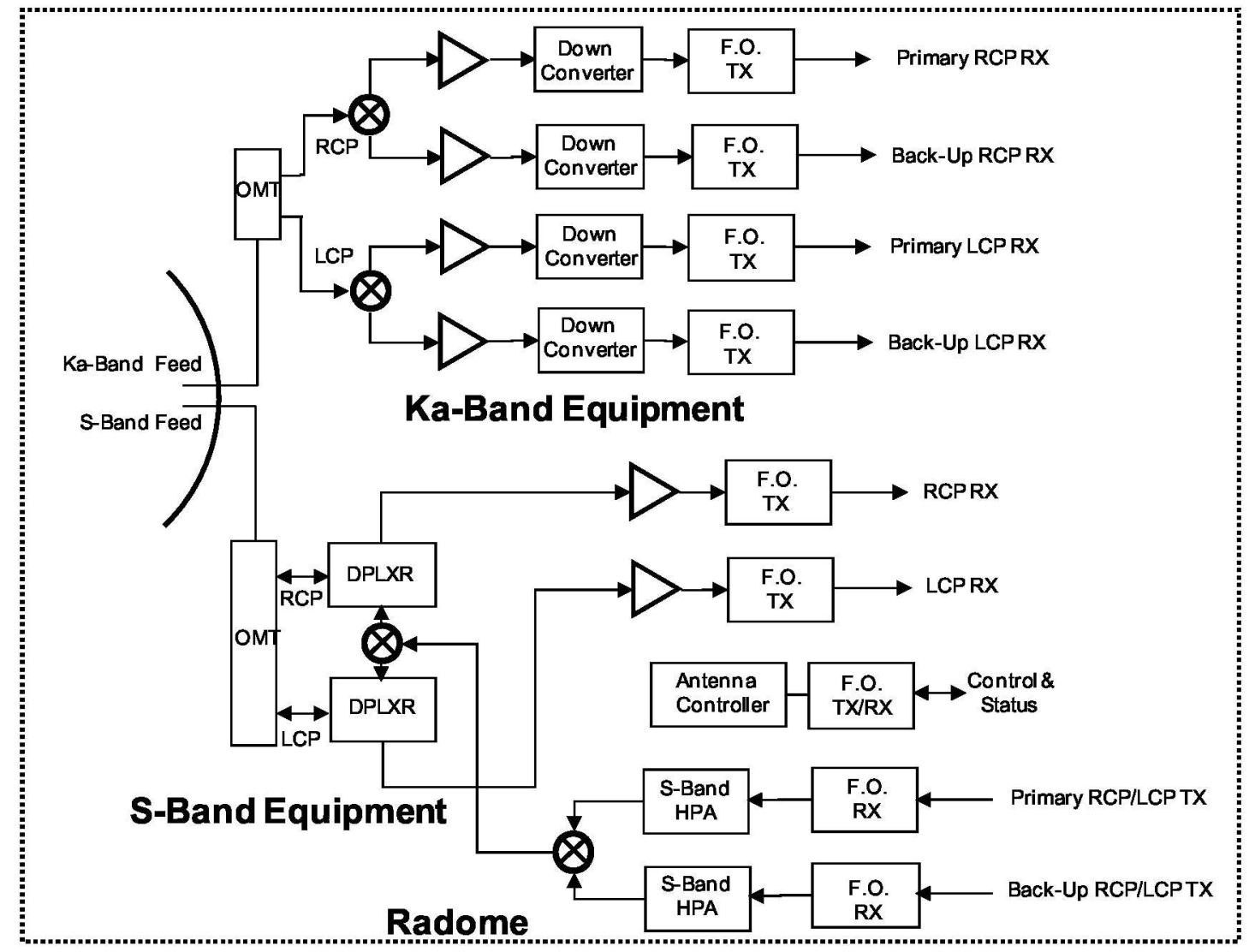

\section{Conclusion (based on RFI responses):}

Three-axis antenna system requirements are reasonable and technically achievable by industry 


\section{Recommended Solution: \\ Wide Area Network}

> NASA Integrated Services Network (NISN) infrastructure will service missions' WAN needs

> Bandwidth requirements for transferring daily data volumes for 3 reference missions studied:

- 490.1 Mbps: minimum needed for transferring expected daily volumes

- 735.1 Mbps: includes recommended $50 \%$ margin

- Margin allows for recovering from data transfer reduction or disruption due to equipment outages, retransmissions, or unforeseen issues

All NEN sites, except for McMurdo, lie on NISN WAN sites, enabling low-cost service by expanding upon existing requirements

> For McMurdo, NASA proposes to refurbish the McMurdo-TDRS Relay System-2 (MTRS-2)

- 300 Mbps-capable ground station

- Data is returned through TDRS to WSC

- Ka-band transmission to TDRS will be needed to support NEN customer missions' high data rates 


\section{Technology Considerations}

Key considerations affecting technology selection:

- Capability

- Maturity

$>$ Spacecraft elements:

- On-board data storage: Multi-terabit space-qualified systems are available

- Coding (error protection): Hardware supporting 600 Msps for each I \& Q channel has been demonstrated

- Compression: Available

- Modulator: Space-qualified high-rate modulator (1.0+ Gbps) forecasted to exist in time for reference missions considered

- Amplifiers (TWTA): Ka-band flight-heritage amplifiers available

- Physical link components: Flight-heritage S- and Ka-band components available

$>$ Ground segment elements:

- Antennas: Ka-band systems (12-14 $\mathrm{m}$ ) available

- Radomes: Available

- SN ground receivers: Planned for procurement (subject to funding)

- NEN ground receivers: 1 Gbps receivers currently commercially available 


\section{Future Considerations}

Technology Insertion

- Selective retransmission, e.g. CCSDS File Delivery Protocol (CFDP)

- LRO experience indicates prudence of protocol-based recovery for weather-induced data losses

- LRO employs CFDP; no science (Ka-band) data lost since June 2009 launch ( $>1700$ LRO supports, $>1800$ hours of communications)

- CCSDS Space Link Extensions (SLE)

- Promotes commonality and international interoperability at the link layer

- Delay/Disruption Tolerant Networking (DTN)

- Enables standardized network layer cross support

$>$ Expansibility Options

- Additional antennas, as needed

- Potential partnerships with other space agencies, commercial providers

- Dual-polarization Ka-band reception (1.5 Gbps per polarization) could provide $3 \mathrm{Gbps}$ downlink

- Single-polarization initial deployment meets currently-known mission requirements and allows deferring investments until needed 


\section{Recommended Solution: Reference Diagram}

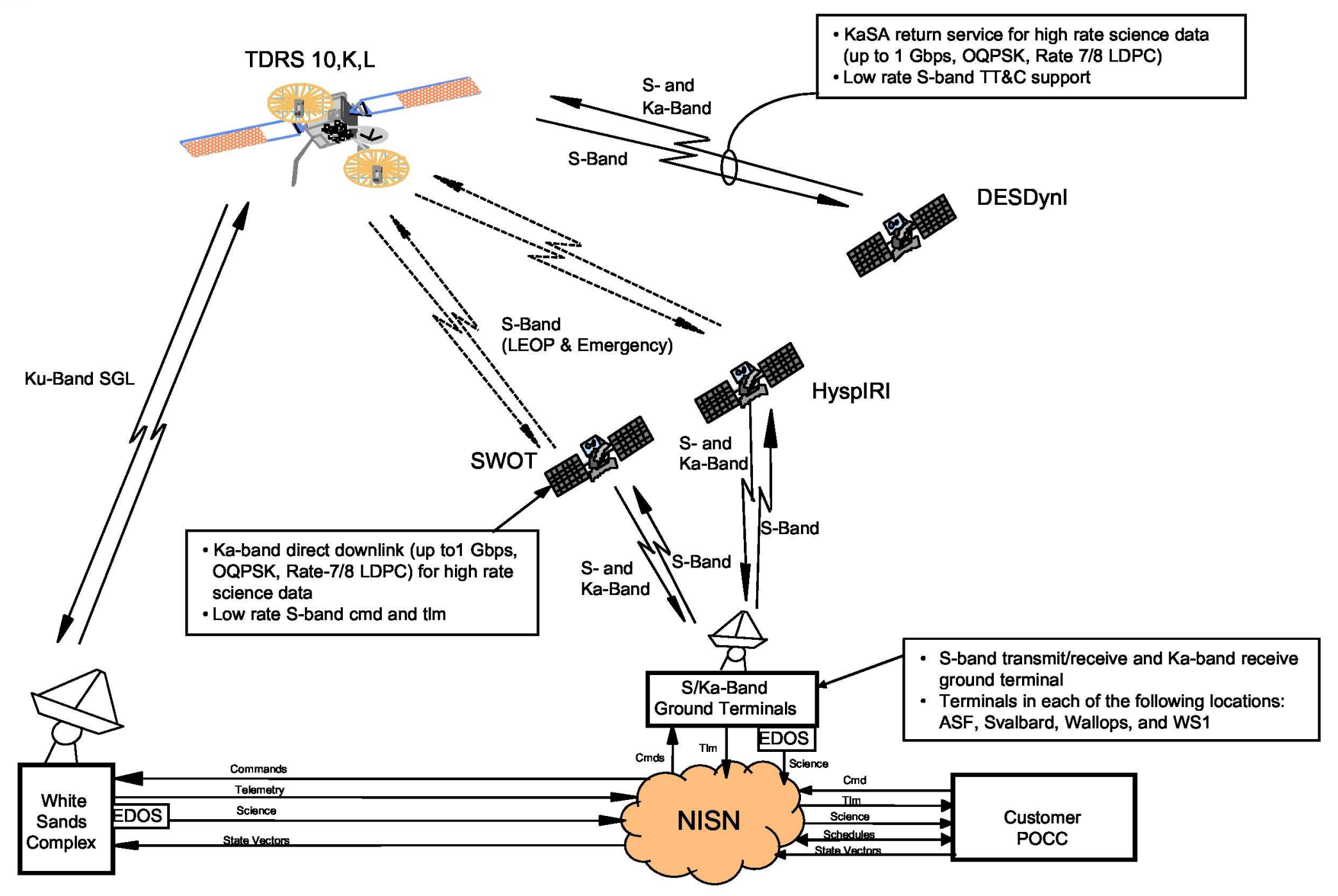

A combination of SN and NEN capabilities will jointly satisfy high-data-rate Ka-band missions 
$>$ Future Earth- and space-science missions will need Ka-band communications to support their extremely high data rates

> SN Ka-band communication feasible today; upgrades to support high data rates are planned

> NEN Ka-band capabilities can be implemented using COTS technology in time to support upcoming high-data-rate missions

> Proposed Ka-band architecture leverages existing assets, where possible, to contain costs

> Continued cooperation between NASA's Science Mission Directorate and SCaN will ensure developing Ka-band communications capabilities to support upcoming spacecraft missions

> The proposed Ka-band network will:

- Enable studying Earth and space phenomena in unprecedented detail

- Help answer scientific questions important to society 
=

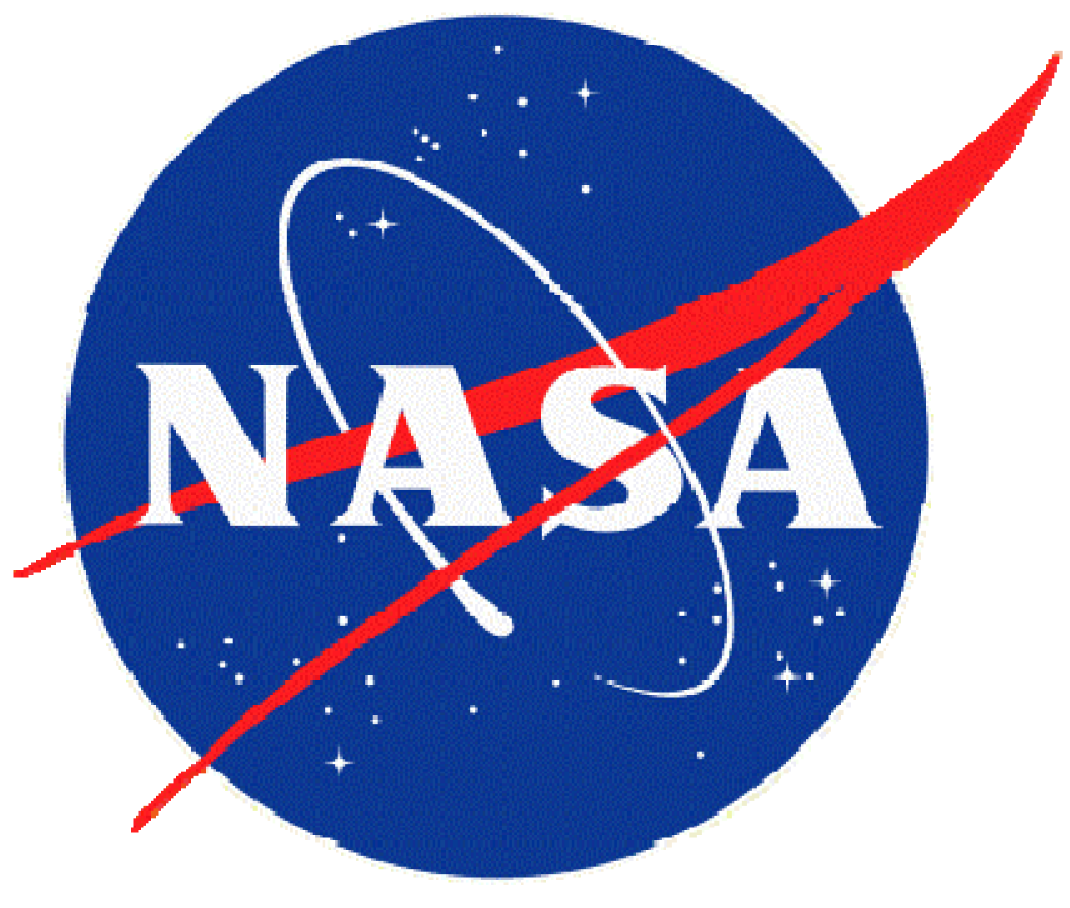

